\title{
SiRNA- Silencing the Culprits
}

\author{
Dr. Neetu Sinha ${ }^{1}$, Dr. Priya Sahni ${ }^{2}$, Dr. Jayadeva $\mathrm{HM}^{3}$, Dr. Shylaja MD ${ }^{4}$, \\ Dr. Pawan Gujjar, Dr. Achala Shenoy ${ }^{6}$
}

\begin{abstract}
Plants as well as animals contain hundreds of small RNAs with distinct genes responsible for their regulation. Any changes in this has corresponding effects on the development and growth of organisms. These small RNAs include microRNA, Piwi-interacting RNA, and short interfering RNA. In case of plants there is complete complimentarity between the microRNA and mRNA, while in animals only partial complimentarity exists. These small RNAs have been used in therapeutics as well as in studying the development process.
\end{abstract}

Keywords: Small RNA, RISC, siRNA, RNAi, Post-transcriptional gene silencing.

\section{Introduction}

Small interfering RNA also known as the silencing RNA or short interfering RNA (siRNA) are a class of double stranded RNA molecules, which have 20-25 base pairs and play a vital role in RNA interference (RNAi). RNA interference is a conserved eukaryotic mechanism in which the double stranded RNA (dsRNA) recognizes homologous mRNAs and causes sequence specific degradation in a multistep process. In the year 1999, David Baulcombe and his group at the Sainsbury Laboratory in Norwich, England reported the presence of siRNAs in plants and described their role in posttranscriptional gene silencing. ${ }^{1}$ Tuschl et al in 2001 experimented with synthetic siRNA inducing RNAi in the mammalian cells. ${ }^{2}$ This pathway is also a potentially powerful tool in several pathogenic cellular pathway. ${ }^{3}$ In the eukaryotes the small RNAs are classified as microRNA (miRNA), Piwi-interacting RNA (piRNA) and siRNA. While the miRNA and piRNA are endogenous in nature siRNA can be both endogenous as well as exogenous. The main differences among these are in the length, structure of precursor molecule, cofactor requirements, modification state, sequence bias as well as regulatory function. These differences vary between species also. ${ }^{4}$

\section{SiRNA}

These small RNAs usually 20-25 base pair long, double stranded with phosphorylated 5' ends and hydroxylated 3' ends along with two overhanging nucleotides, was originally observed in transgene- and virusinduced silencing in plants. They are synthesized from the long dsRNAs convergent mRNA transcripts, natural sense-antisense pairs, duplexes involving pseudogene derived from antisense transcriptions and small hairpin RNAs (hpRNA). Centromeres, transposons and other repetitive sequences also act as source of these small interfering RNAs. ${ }^{5}$

\section{RISC}

RNA-induced silencing complex (RISC) is a multi-subunit protein complex which seeks an appropriate target mRNA to which the siRNA attaches, unwinds and its antisense directs the degradation of the complementary strand of the mRNA by using a combination of exo and endonuclease enzymes. The si-RISC assembly pathway requires three proteins- Dicer, TRBP and Ago 2. This trimer being called as the RISCLoading Complex (RLC). ${ }^{6}$

\section{RNAi}

RNA interference also known as siRNA silencing or siRNA knockdown, as introduced by Fire and Mello refers to the introduction of a homologous double stranded RNA to specifically target a gene's product, resulting in a null or hypomorphic phenotypes.It is a highly specific system for gene manipulation and highly potent too. Previously this phenomena was also known as co-suppression, in fungi as quelling, in plants as posttranscriptional gene silencing (PTGS), and in nematodes as RNAi. ${ }^{7}$ RNAi is an intracellular process which converts the precursor dsRNA into functional siRNA. ${ }^{8}$ As natural siRNAs are formed by endogenous pathway, the introduction of synthetic siRNAs can be utilised for targeting genes for both research as well as therapeutics. ${ }^{9}$ As seen in plants, RNAi protects cells from viral infection and inappropriate expression of repetitive sequences and transposable elements. ${ }^{10}$ In mammalian cells siRNA are used to trigger RNAi for intentional reduction in the expression of certain genes. ${ }^{11}$ 


\section{In Therapeutics}

The specificity of siRNAs has made them a potential and attractive candidate for targeting dominant negative mutations that affect single alleles. The selective knockdown of defective genes without affecting the wildtype copy, has the potential to ameliorate the phrnotypic expressions of a significant number of genetic, oncological and neurological disorders. ${ }^{12-15}$

In the field of medical therapeutics, the siRNAs have been used successfully in phase I clinical trials for Age-related Macular Degeneration (AMD) ${ }^{16}$ Potential antiviral therapies utilising these small RNAs are the use of topical microbicide against herpes simplex virus type 2 . Viral gene expressions have been inhibited in cancerous cells, host receptors and coreceptors for HIV have been knockdown, Genes for Hepatitis A and hepatitis B, as well as influenza virus have been silenced, and siRNAs have also been used to inhibit the replication of measles virus. ${ }^{17}$ In phase I clinical trials on patients with advanced cancer metastasized to liver, siRNA were delivered through lipid nanoparticles, targeting genes coding for Vascular Endothelial Growth Factor (VEGF) and Kinesin spindle protein (KSP). The results showed either stabilization or regression of metastasis. Pharmacodynamic analysis of the biopsy specimens obtained from these patients revealed that the molecules had reached the intended target. ${ }^{18,19}$ Proof of concept trials using siRNA as post exposure prophylaxis against Ebola virus, in non-human primates has shown $100 \%$ efficacy. $100 \%$ of the subjects survived a lethal dose of Zaire Ebola virus. There have been extensive efforts in computational biology directed towards the design of a successful reagent that maximizes gene knockdown but minimizes 'off-target effects'. The study of human genomes has shown that about $10 \%$ of possible siRNAs have substantial off- target effects. ${ }^{21}$ In case of Mantle cell lymphoma (MCL), an incurable B cell non-Hodgkin's lymphoma, characterized by $\mathrm{t}(11 ; 14)(\mathrm{q} 13 ; \mathrm{q} 32)$ translocation causes juxtaposition of proto-oncogene CCND1, encoding for cyclin D1. Cyclin D1 is a regulator of cell cycle progression hence knocking down cyclin D1 using siRNA resulted in significant growth retardation, cell cycle arrest as well as induction of apoptosis. ${ }^{22}$

\section{Conclusion}

The potential for utilization of siRNAs as a vehicle for targeting diseases and defects involving heterogenous mutations is the future of research in genomics.

\section{References}

[1]. Hamilton A, Baulcombe D. A species of small antisense RNA in posttranscriptional gene silencing in plants. Science. 1999. 286(5441);950-2.

[2]. Elbashir S, Harborth J, Lendeckel W, Yalcin A, Weber K, Tuschl T. Duplexes of 21-nucleotide RNAs mediate RNA interference in cultured mammalian cells. Nature. 2001. 411(6836); 494-988.

[3]. Aagaard L, Rossi JJ. RNAi therapeutics : principles, prospects and challenges.Advanced Drug Delivery Reviews. 2007. 59(2-3); 75-86.

[4]. Kim VN. Small RNAs : classification, biogenesis and function. Molecules and cells. 2005.19(1); 1-15

[5]. Bernstein E, Caudy A, Hammond S, Hannon G. Role for a bidentate ribonuclease in the initiation step of RNA interference. Nature. 2001. 409(6818); 363-6

[6]. Daneholt B. Advanced information: RNA interference. The Novel prize in Physiology or Medicine. 2006.

[7]. Fire A, Xu S, Montgomery MK, Kostas SA, Driver SE, Mello CC. Potent and specific genetic interference by double stranded RNA in Caenorhabditis elegans. Nature.1998. 391; 806-811.

[8]. Novina CD, Sharp PA. The RNAi revolution. Nature. 2004. 430; 161-164

[9]. Sullivan CS, Ganem D. A virus encoded inhibitor that blocks RNA interference in mammalian cells. J Virol. 2005. 79; 7371 -7379.

[10]. Bennasser Y, Le SY, Benkirane M, Jeang KT. Evidence that HIV-1 encodes an siRNA and a suppressor of RNA silencing. Immunity. 2005.22; 607-619.

[11]. Cullen BR. Is RNA interference involved in intrinsic antiviral immunity in mammals ? Nat Immunol. 2006. 7; 563-567.

[12]. Brummelkamp TR, Bernards R, Agami R. Stable suppression of tumorigenicity by virus mediated RNA interference. Cancer Cell. 2002. 2; 243-247.

[13]. Ding H, Schwarz DS, Keene A, Affar el B, Fenton L, Xia X et al. Selective silencing by RNAi of a dominant allele that causes amyotrophic lateral sclerosis. Aging Cell. 2003. 2;209-217.

[14]. Gonzalez-Alegre P, Bode N, Davidson BL, Paulson HL. Silencing primary dystonia: lentiviral mediated RNA interference therapy for DYT1 dystonia. J Neurosci. 2005. 25; 10502-10509.

[15]. Miller VM, Xia H, Marrs GL, Gouvion CM, Lee G, Davidson BL et al. Allele specific silencing of dominant disease genes. Proc Natl Acad Sci USA. 2003. 100; 7195-7200.

[16]. Tansey B. Macular degeneration treatment interferes with RNA messages. 2006. San Fransisco Cronicle.

[17]. Whitehead KA, Dahlman JE, Langer RS, Anderson DG. Silencing or Stimulation ? siRNA delivery and the immune system. Annual Review of Chemical and Biomolecular Engineering. 2011. 2;77-96.

[18]. Vall D' Hebron Institute of Oncology. First-in-man study demonstrates the therapeutic effect of RNAi gene silencing in cancer treatment. 2013.

[19]. Tabernero J, Shapiro G, Lorusso P, Cervantes A, Schwartz G, Weiss G et al.First-in-humans trial of an RNA interference therapeutic targeting VEGF and KSP in cancer patients with liver involvement. Cancer Discovery. 2013. 3(4);406-417.

[20]. Geisbert TW, Lee ACH, Robbins M, Geisbert JB, Honko AN, Sood V, Hensley LE, MacLachlan I. Postexposure protection of nonhuman primates against a lethal Ebola virus challenge with RNA interference : a proof of concept study. The Lancet. 2010. 375(9729);1896-1905.

[21]. Du Q, Thornberg H, Wang J, Wahlestedt C, Liang Z. A systematic analysis of the silencing effects of an active siRNA at all single nucleotide mismatched target sites. Nucleic Acids Res. 2005.33; 1671-1677.

[22]. Weinstein S, Emmanuel R, Jacobi AM, Abraham A, Behlke MA, et al. RNA inhibition highlights Cyclin D1 as a potential therapeutic target for mantle cell lymphoma. Plos One. 2012. 7(8); e43343. 